This is a postprint version of the following published document:

Prior Cano, E.; Dios Fernández, C. de; Criado Serrano, A.R.; Ortsiefer, M.; Meissner, P. and Acedo, P. (2014). Experimental study of VCSEL-based optical frequency comb generators. IEEE phtonics technology letters, 26 (21), pp. $2118-2121$

DOI: 10.1109/LPT.2014.2348594

(C) 2014 IEEE. Personal use of this material is permitted. Permission from IEEE must be obtained for all other uses, in any current or future media, including reprinting/republishing this material for advertising or promotional purposes, creating new collective works, for resale or redistribution to servers or lists, or reuse of any copyrighted component of this work in other works. 


\title{
Experimental Study of VCSEL-Based Optical Frequency Comb Generators
}

\author{
Estefanía Prior Cano, Cristina de Dios Fernandez, Ángel Rubén Criado Serrano, \\ Markus Ortsiefer, Peter Meissner, and Pablo Acedo, Member, IEEE
}

\begin{abstract}
In this letter, we explore the performance of an optical frequency comb generator (OFCG) sources based on vertical-cavity surface-emitting laser diodes. The direct gain switching (GS) technique, the indirect electro-optical (EO) technique, and a combination of both have been experimentally evaluated. We have observed that this later combination gives birth to an enhanced OFCG that offers a tunable improved comb in terms of frequency span, flatness, and coherence with respect to the sole EO approach, as it partly inherits the high-efficiency qualities of the GS-OFCG while offering a wider span.
\end{abstract}

Index Terms-Optical frequency comb generator, VCSEL, gain switching, phase modulator, electro-optical comb.

\section{INTRODUCTION}

$\mathbf{T}$ HE interest Optical Frequency Comb Generators (OFCG) have attracted in the last decade is a consequence of the versatility they cherish. This equally spaced group of optical modes has found application in a large variety of fields. Spectroscopy [1], optical communications [2], THz generation [3], optical arbitrary waveform generation [4], Metrology [5], or microwave photonic filters [6] are some examples of entire disciplines enjoying the solutions that the use of OFCG can provide. To cover such a wide range of fields and expand it an OFCG should offer a tunable, wide and flat optical comb. Its modes should also be highly correlated, since this is an important requirement for applications such as $\mathrm{THz}$ generation [3]. An ideal comb should cover these features while taking care of compactness, energy and cost efficiency.

OFCG sources based on lasers diodes (LD) are good candidates to meet the abovementioned requirements, especially if they can use Cost of the Shelf (COTS) devices. The maturity of commercially available LD would benefit the resulting OFCG taking it from the laboratory to the application field in a reliable and cost efficient way. Several techniques have already demonstrated promising results [7], [8]. They can be grouped in direct or indirect techniques.

Manuscript received July 18, 2014

E. P. Cano, C. de Dios Fernandez, and P. Acedo are with the Department of Electronics Technology, Universidad Carlos III de Madrid, Madrid 28911, Spain (e-mail: eprior@ing.uc3m.es; cdios@ing.uc3m.es; pag@ing.uc3m.es).

Á. R. C. Serrano is with Luz WaveLabs, Madrid 28919, Spain (e-mail: ruben.criado@luzwavelabs.com).

M. Ortsiefer is with Vertilas $\mathrm{GmbH}$, Munich 85748, Germany (e-mail: ortsiefer@vertilas.com).

P. Meissner is with the Technische Universität Darmstadt, Darmstadt 64289 , Germany (e-mail: meissner@imp.tu-darmstadt.de).

Color versions of one or more of the figures in this letter are available online at http://ieeexplore.ieee.org
Direct OFCG techniques are those where the generation of the comb occurs within the diode laser itself when it is operating under pulsed regime. One of the most interesting approaches is Gain Switching (GS). It is based on large signal modulation of the current injected to the LD and can be applied to any COTS device [9]. It offers tunability of the frequency spacing of the comb and a strong phase relation between its modes [3]. Still, optical span and flatness reported so far need to be improved to make GS-OFCG a high quality alternative.

Indirect OFCG techniques process the light after it is emitted using a nonlinear optical element. The most extended approach is the use of external Electro Optical modulators (EO-OFCG) [6], [8]. The resulting comb is tunable and can offer a span in the $\mathrm{THz}$ range using a cascade of modulators that requires a careful management of the RF power and the phase of the electrical and the optical signals all along the set-up. This technique typically uses other laser sources but it can also be applied to commercially available LD devices.

There exists a third way to generate optical combs using diode laser sources and it is based on the combination of direct and indirect techniques. A Combined-OFCG source based on GS and EO modulators [3], [10] permits the enhancement of the initial GS comb span and its flatness.

But the characteristics of the final comb are not only determined by the technique. The diode laser technology plays a major role. The use of edge emitting technologies such as Fabry-Perot [10], Distributed Feedback Bragg lasers or Discrete Mode [11] has been recently reported. In this sense, our previous work [7] shows that the use of high performance Vertical Cavity Surface Emitting Lasers (VCSEL) permits the generation of an enhanced comb, record in energy efficiency and optical span. This result drives our interest to study the possibilities of this technology as the core of an OFCG that could meet the requirements detailed before. In the present work, we systematically evaluate the performance of VCSELs for OFCG based on lasers diodes. We have explored the use of direct GS-OFCG, indirect EO-OFCG and Combined-OFCG techniques and compared the resulting combs in terms of span, flatness, frequency tunability, efficiency and coherence.

\section{EXPERIMENTAL SET-UP}

The core of our experimental scheme is a state of the technology VCSEL diode laser (VERTILAS VL-1550-8G-P2$\mathrm{H} 4, \mathrm{I}_{\mathrm{th}}=2.9 \mathrm{~mA}$ ). It is mounted on a pre-commercial prototype developed in collaboration with VERTILAS that includes the 

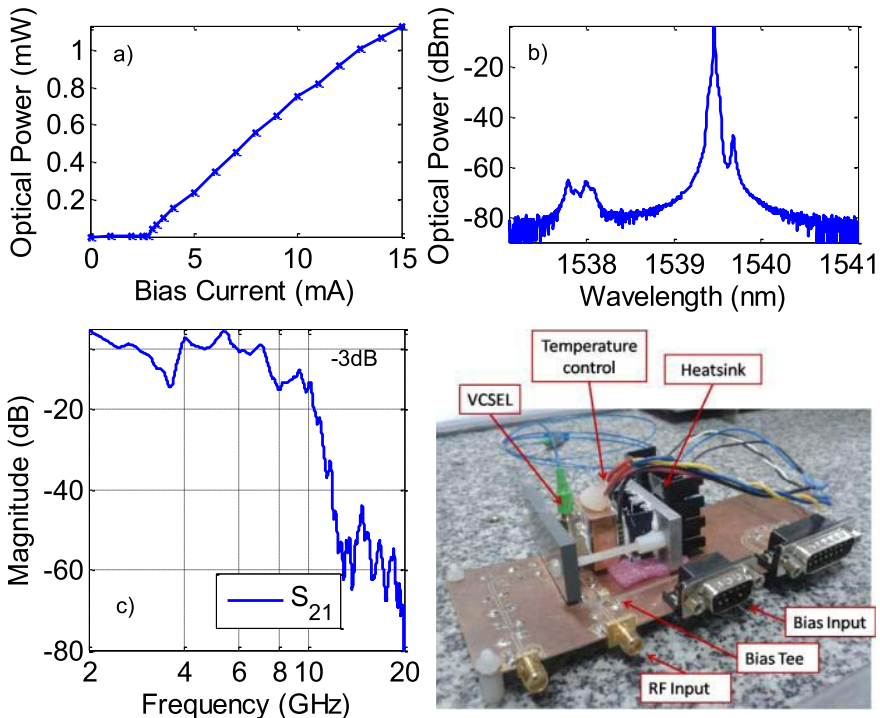

Fig. 1. Static and dynamic characterization of the VCSEL device. a) IP optoelectronic curve. b) Continuous wave optical spectra and c) dynamic small signal response with $\mathrm{S} 21$ scattering parameter at $\mathrm{I}_{\text {bias }}=11.4 \mathrm{~mA}$. The picture shows the VCSEL device pre-commercial prototype.

VCSEL adapted for radiofrequency (RF) modulation with a specially designed high frequency matching PCB network. An optical isolator is included to avoid optical feedback. Its temperature is stabilized at $25^{\circ} \mathrm{C}$. A typical characterization of the device is depicted in Figure 1. At the $\mathrm{I}_{\text {bias }}=11.4 \mathrm{~mA}$ considered (best GS performance) the electronic $3 \mathrm{~dB}$ bandwidth of the VCSEL is $7 \mathrm{GHz}$. Nevertheless, the frequency response does not show a perfect flat low pass shape due to impedance mismatches related to the high frequency $\mathrm{PCB}$ prototype response (3-4 GHz range).

In Figure 2 we show the set-up developed for the present work. It consists of two different optical subsystems. The first one is the direct Gain Switching Optical Frequency Comb Generator (GS-OFCG). When the GS regime is induced, the injected RF power into the laser is $P_{R F, G S}=15 \mathrm{dBm}$ and the bias current is $\mathrm{I}_{\mathrm{bias}}=11.4 \mathrm{~mA}$ at $\mathrm{f}_{\mathrm{RF}}=5 \mathrm{GHz}$. These working conditions were chosen in order to offer the best OFCG performance [7].

The second subsystem is the indirect Electro-Optical generation scheme (EO-OFCG) also shown in Figure 2. It is composed of the VCSEL diode in continuous wave $(\mathrm{CW})$ operation (no RF modulation to the diode laser) and an external Electro-Optic modulation stage. In this letter this stage consists of two standard phase modulators ( $3 \mathrm{~dB}$ bandwidth up to $10 \mathrm{GHz}$ ) in cascade configuration. This scheme is the core of larger architectures that have successfully produced enhanced optical frequency combs [6], [8]. As the bias conditions of the VCSEL do not affect the EO stage, we also use $\mathrm{I}_{\text {bias }}=11.4 \mathrm{~mA}$ to facilitate the comparison. An RF power of $\mathrm{P}_{\mathrm{RF}, \mathrm{EO}}=28 \mathrm{dBm}$ is needed for each external modulators to work under optimum conditions (wider span of the resulting comb). Hence, a high power RF amplifier is included. Phase matching between this modulating signals and the optical signal entering the modulators is critical, so a phase shifter is needed before their $\mathrm{RF}$ inputs and has to be tuned to optimize the resulting comb.

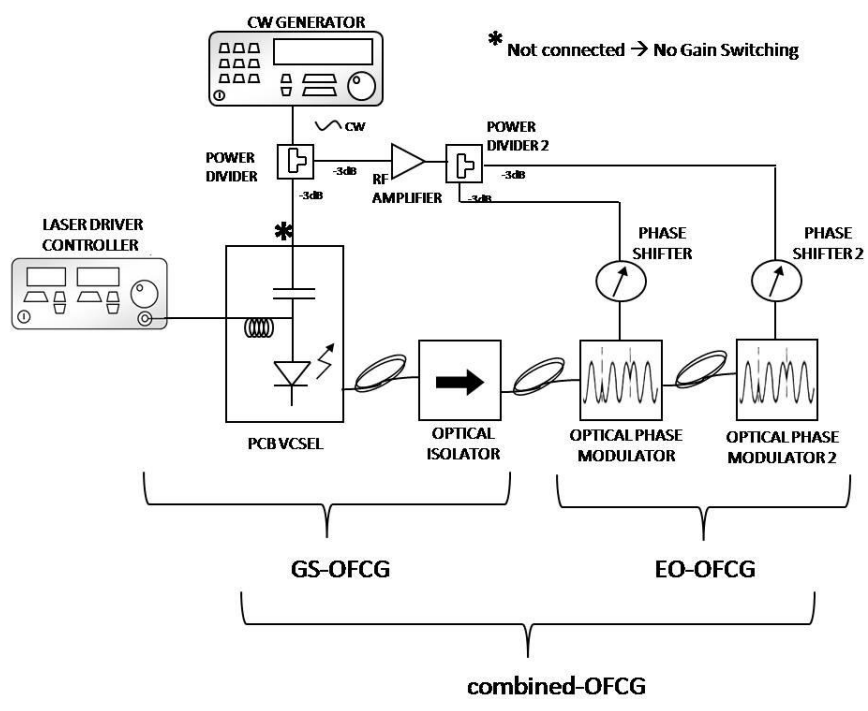

Fig. 2. The experimental set-up consists of two subsystems. Left: GS-OFCG based on a VCSEL source under Gain Switching. Right: EO-OFCG based on two optical Phase Modulators. The complete set-up is the Combined-OFCG that connects both subsystems in cascade linking them with the same RF modulating signal.

The simultaneous operation of the two subsystems described before permits the experimental evaluation of the CombinedOFCG technique using VCSELs. The same RF signal is split to induce the GS regime in the direct comb subsystem with $\mathrm{P}_{\mathrm{RF}, \mathrm{GS}}=15 \mathrm{dBm}$ and to modulate each modulator of the indirect EO-OFCG with $\mathrm{P}_{\mathrm{RF}, \mathrm{EO}}=28 \mathrm{dBm}$, bounding the subsystems together.

\section{RESULTS}

In this section, we compare the optical combs we have obtained based on state of the technology VCSEL device. We have used the three available techniques to develop an OFCG based on a laser diodes: direct GS, indirect EO and the combination of both. We have evaluated the comb span, its flatness, its frequency tunability, the phase relation between its modes and the energy efficiency of the OFCG sources.

\section{A. Optical Frequency Span and Flatness}

For applications such as $\mathrm{THz}$ photonic generation, the useful optical span can be defined up to 20dB [3]. For optical communications, the flatness of the comb is more critical and the useful modes lie within a $3 \mathrm{~dB}$ or $10 \mathrm{~dB}$ bandwidth [11]. This is why we have evaluated the comb span using several metrics. We have also defined the comb flatness as the ratio of the $3 \mathrm{~dB}$ to the $10 \mathrm{~dB}$ bandwidth and the $10 \mathrm{~dB}$ to the $20 \mathrm{~dB}$ span. The closer these values are to 1 , the closer the comb is to exhibit a flat-top shape.

In Figure 3.a we show the VCSEL based GS optical comb with $\mathrm{f}_{\mathrm{RF}}=5 \mathrm{GHz}$. The output of the VCSEL has a $3 \mathrm{~dB}$ bandwidth of $80 \mathrm{GHz}$ (16 modes) and a $20 \mathrm{~dB}$ bandwidth of $106 \mathrm{GHz}$ (21 optical modes). The comb exhibits a remarkable flatness (0.84 and 0.9 in Table I). Figure 3.b shows the comb obtained using the indirect EO-OFCG technique alone with the same $f_{R F}=5 \mathrm{GHz}$. The $3 \mathrm{~dB}$ bandwidth reaches only 

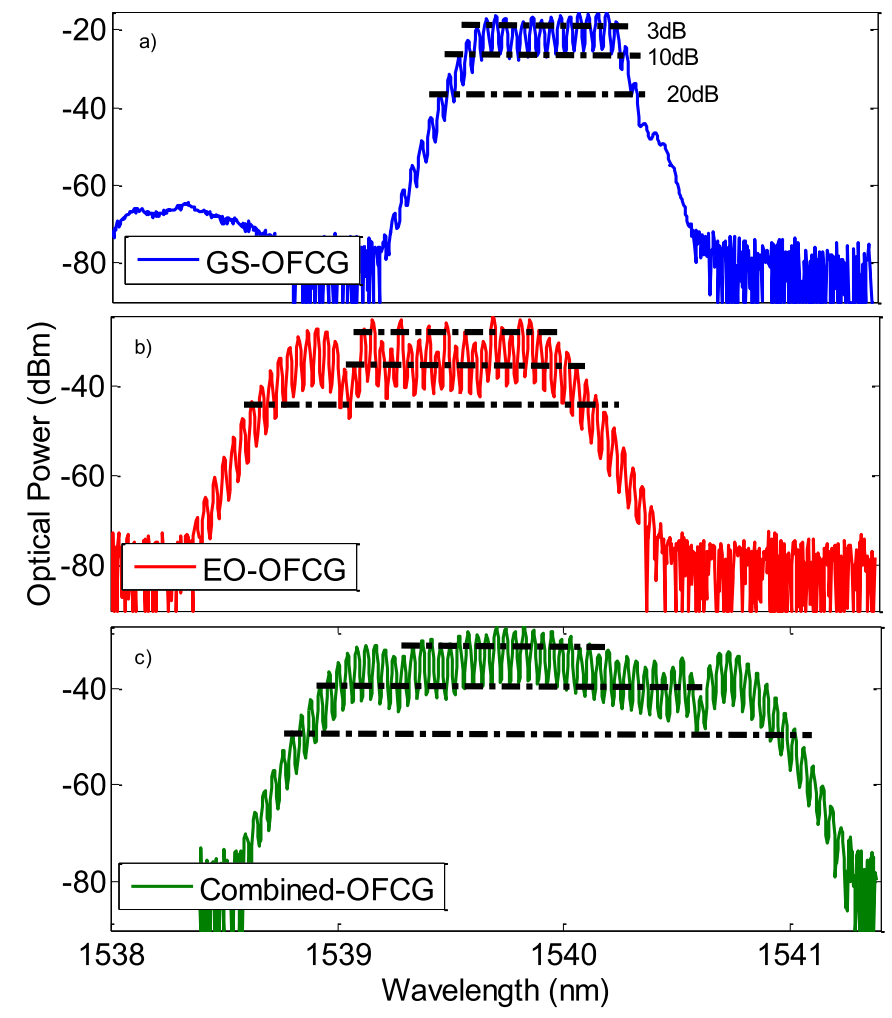

Fig. 3. OFCG techniques with VCSEL diode laser: comparison. a) GS-OFCG technique. b) EO-OFCG. c) Combined-OFCG. The modulation frequency is $\mathrm{f}_{\mathrm{RF}}=5 \mathrm{GHz} .3 \mathrm{dBm}, 10 \mathrm{~dB}$ and $20 \mathrm{~dB}$ bandwidths of the combs are indicated. The combined-OFCG has a $20 \mathrm{~dB}$ span of $272 \mathrm{GHz}$ and 53 lines.

TABLE I

COMPARISON DIRECT, INDIRECT AND COMBINED OFCG: FREQUENCY SPAN AND FLATNESS @ $\mathrm{f}_{\mathrm{RF}}=5 \mathrm{GHz}$

\begin{tabular}{|c|c|c|c|}
\hline Metric & GS-OFCG & EO-OFCG & Combined-OFCG \\
\hline Span@3dB & $\begin{array}{c}80 \mathrm{GHz} \\
16 \text { modes }\end{array}$ & $\begin{array}{c}16 \mathrm{GHz} \\
3 \text { modes }\end{array}$ & $\begin{array}{c}100 \mathrm{GHz} \\
20 \text { modes }\end{array}$ \\
\hline Span@10dB & $97 \mathrm{GHz}$ & $126 \mathrm{GHz}(165)$ & $208 \mathrm{GHz}(248)$ \\
\hline Flatness & 19 modes & 25 modes (33) & 41 modes (49) \\
\hline $\begin{array}{c}3 \mathrm{~dB} / 10 \mathrm{~dB} \\
\mathrm{f} 3 \mathrm{~dB}\end{array}$ & $16 / 19=0.84$ & $3 / 25=0.12$ & $20 / 41=0.48$ \\
\hline Span@20dB & $106 \mathrm{GHz}$ & $193 \mathrm{GHz}$ & $272 \mathrm{GHz}$ \\
\hline Flatness & 21 modes & 38 modes & 53 modes \\
\hline $\begin{array}{c}10 \mathrm{~dB} / 20 \mathrm{~dB} \\
\mathrm{f} 10 \mathrm{~dB}\end{array}$ & $19 / 21=0.9$ & $25 / 38=0.66$ & $41 / 53=0.77$ \\
\hline
\end{tabular}

$16 \mathrm{GHz}$ ( 3 modes), while its $20 \mathrm{~dB}$ bandwidth rises to $193 \mathrm{GHz}$. The flatness of the EO comb is far from 1 ( 0.12 and 0.66 Table I). To improve the flatness of this type of comb an extra External Intensity Modulator is typically included. Figure 1.c depicts the Combined-OFCG comb that has a $3 \mathrm{~dB}$ span of $100 \mathrm{GHz}$ ( 20 modes) and a $20 \mathrm{~dB}$ span of $272 \mathrm{GHz}$ ( 53 modes).

We have seen that the VCSEL based comb using GS technique offers the best flatness but a limited optical span. An increased $20 \mathrm{~dB}$ span can be achieved with the EO scheme, however, the flatness of the comb is compromised and extra
TABLE II

COMPARISON DIRECT, INDIRECT AND COMBINED OFCG: TUNABILITY

\begin{tabular}{cccc}
\hline \hline $\begin{array}{c}\mathrm{f}_{\mathrm{RF}}(\mathrm{GHz}) / \\
\text { Span@20dB }\end{array}$ & GS-OFCG & EO-OFCG & Combined-OFCG \\
\hline \hline \multirow{2}{*}{5} & $106 \mathrm{GHz}$ & $193 \mathrm{GHz}$ & $272 \mathrm{GHz}$ \\
& 21 modes & 38 modes & 53 modes \\
\hline \multirow{2}{*}{9} & $58 \mathrm{GHz}$ & $179 \mathrm{GHz}$ & $206 \mathrm{GHz}$ \\
& 7 modes & 19 modes & $23 \mathrm{modes}$ \\
\hline \multirow{2}{*}{12} & 1 mode & $297 \mathrm{GHz}$ & $297 \mathrm{GHz}$ \\
& (No GS) & 24 modes & 24 modes \\
\hline
\end{tabular}

external modulating elements need to be included in the system. However, the Combined approach applied to the VCSEL device offers an enhanced optical span for all the metrics (40\% wider than the EO-OFCG and $150 \%$ wider that the GS at $20 \mathrm{~dB}$ ) and a flatness that is below the direct GS approach but better than the indirect $\mathrm{EO}$ without adding additional elements.

\section{B. Frequency Tunability}

The OFCG techniques considered in this letter have the possibility to offer an optical comb that can change the frequency separation between its modes by changing the frequency of the RF modulating signal, $f_{R F}$. But, for different $f_{R F}$, the characteristics of the resulting combs may vary. In Table II we present the comb span and the number of modes at $20 \mathrm{~dB}$ for different $f_{R F}$.

When the $\mathrm{f}_{\mathrm{RF}}$ lies within the electronic bandwidth of the VCSEL device (4 to $7 \mathrm{GHz}$ for $\mathrm{I}_{\text {bias }}=11.4 \mathrm{~mA}$, see figure 1.c), the GS-OFCG and the Combined-OFCG offer their best performance. When the $f_{R F}$ increases further, the VCSEL does not enter Gain Switching operation and the GS and Combined combs offer spans significantly smaller. For $f_{R F}>12 \mathrm{GHz}$, the GS comb is inexistent. Then, the frequency tunability for the GS and combined combs is only possible within the electronic bandwidth of the laser diode. This means that careful choice of the parameters that define the Gain Switching regime ( $\mathrm{I}_{\text {bias }}$, $\mathrm{f}_{\mathrm{RF}}, \mathrm{P}_{\mathrm{RF}, \mathrm{GS}}$ ) has to be carried out in order to manage the optical span and/or the number of modes as $f_{R F}$ changes.

For the EO-OFCG, there exists an optical comb for all the $\mathrm{f}_{\mathrm{RF}}$ under study, but the available span and/or number of modes also depends on the $f_{R F}$. The optical span and the number of modes decrease at $9 \mathrm{GHz}$ to increase again at $12 \mathrm{GHz}$. If a constant span or number of modes is needed, the $\mathrm{P}_{\mathrm{RF}, \mathrm{EO}}$ modulating the $\mathrm{EO}$ devices and the phase matching between the RF and optical signals should also be changed as $f_{R F}$ increases.

\section{Coherence of the Modes of the Comb}

We have studied the coherence between the modes of the combs obtained evaluating the phase noise of the beat tone generated after mixing its modes. A degradation of the phase noise of this beat tone is associated to an overall loss of coherence among the optical modes. In [7] we found that the phase noise of the VCSEL based GS-OFCG is similar to that of the reference signal. In the present work (Figure 4) we have evaluated EO and Combined techniques. 


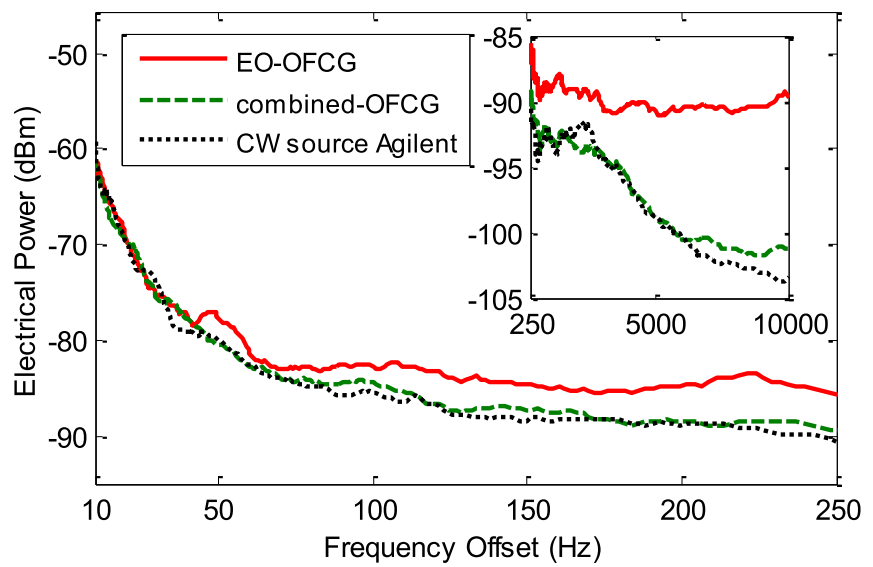

Fig. 4. Phase Noise of the electrical signal detected at the output of the setup: signal generated after the EO-OFCG (solid), after the Combined-OFCG (dashed) and the signal of the RF source (dotted). The beat-tone obtained from the Combined-OFCG has the same Phase Noise as the source. The signal is degraded with respect to the RF source for the EO-OFCG. The complete signal is shown in the inset while a zoom is in the main picture.

As we can see from this Fig. 4, the phase noise of the VCSEL-based Combined-OFCG and the indirect EO-OFCG remain similar up to $40 \mathrm{~Hz}$ frequency offset. From this point on, the Combined source exhibits a phase noise that is up to $11 \mathrm{~dB}$ less that the phase noise of the indirect $\mathrm{EO}$ beat tone (at $10 \mathrm{KHz}$ offset frequency). Also, the phase noise of the Combined-OFCG remains close to the phase noise of the modulating RF source up to $6.2 \mathrm{KHz}$. From that point on, they slightly diverge and a maximum difference of $2.2 \mathrm{~dB}$ is observable at $9.7 \mathrm{KHz}$. We have to note here that around $2.2 \mathrm{KHz}$, the phase noise of the RF signal exceeds that of the Combined beat tone in $1.6 \mathrm{~dB}$. This can be attributed to instabilities in the radio-frequency generator. In conclusion, the Combined-OFCG offers a comb whose mode coherence and stability are linked to the initial RF modulating source and exceeds that of the EO approach.

\section{Energy Efficiency}

If we consider the RF power consumption of the different schemes and compare it with the number of generated modes at $20 \mathrm{~dB}$, we find that the direct GS approach consumes $15 \mathrm{dBm} / 21$ modes $=1.5 \mathrm{~mW} /$ mode. The EO-OFCG needs $33.1 \mathrm{~mW}$ per mode while the combined approach utilizes $24.4 \mathrm{~mW} /$ mode. The peak optical power of the generated modes within the $3 \mathrm{~dB}$ bandwidth is $-16 \mathrm{dBm}$ for the direct GS comb, $-25 \mathrm{dBm}$ for the indirect $\mathrm{EO}$ and $-28 \mathrm{dBm}$ for the Combined-OFCG.

\section{CONCLUSiOnS}

After evaluating the results we have presented we can find that the Combined VCSEL-based OFCG offers better results in terms of available optical span, number of modes and coherence of the generate modes. The direct GS VCSELbased OFCG exhibits also some extra advantages, like the best flatness and the best energy efficiency. The indirect EO-OFGC using VCSEL does not excel in any of the parameters evaluated. Of course its span or flatness could be improved by adding an extra element or choosing EO modulators with better performance, but that would also improve the characteristics of the combined approach, leaving the comparison unchanged. All the techniques are tunable, being the EO the one that offers a higher frequency shift. In all the cases, to generate a stable comb in terms on the number of modes or the span, other parameters that define the working point of the OFCG have to be carefully adjusted as the frequency changes.

Then, the generation of optical combs based on VCSEL diode lasers offers better results when the GS and the EO techniques are combined: the advantages of both techniques add up to offer a better comb in all of the aspects evaluated. Only the energy efficiency decreases with respect to the GS generation. If we compare the EO and Combined techniques in terms of experimental complexity, the combined approach is the most indicated, since its set-up is equivalent to the EO, and the performance of the final comb is clearly enhanced.

If we compare VCSEL based OFCG with sources based on other laser diode technology [10], [11], we find that with equivalent set-ups, VCSELs offer wider combs with better energy efficiency.

Special attention deserves the results describing the coherence of the modes within the generated combs. GS and Combined techniques offer a comb with high stability linked to the CW RF source considered. This is a new remarkable result whose understanding and exploitation will center or future efforts, giving its importance for applications like $\mathrm{THz}$ or mid-IR generation.

\section{REFERENCES}

[1] F. Zhu, T. Mohamed, J. Strohaber, A. A. Kolomenskii, T. Udem, and H. A. Schuessler, "Real-time dual frequency comb spectroscopy in the near infrared," Appl. Phys. Lett., vol. 102, no. 12, p. 121116, 2013.

[2] P. J. Delfyett et al., "Optical frequency combs from semiconductor lasers and applications in ultrawideband signal processing and communications," J. Lightw. Technol., vol. 24, no. 7, pp. 2701-2719, Jul. 2006.

[3] A. R. Criado et al., "Continuous-wave sub- $\mathrm{THz}$ photonic generation with ultra-narrow linewidth, ultra-high resolution, full frequency range coverage and high long-term frequency stability," IEEE Trans. Terahertz. Sci. Technol., vol. 3, no. 4, pp. 461-471, Jul. 2013.

[4] S. T. Cundiff and A. M. Weiner, "Optical arbitrary waveform generation," Nature Photon., vol. 4, no. 11, pp. 760-766, Oct. 2010.

[5] T. Udem, R. Holzwarth, and T. W. Hänsch, "Optical frequency metrology," Nature, vol. 416, no. 6877, pp. 233-237, Mar. 2002.

[6] R. Wu, C. M. Long, D. E. Leaird, and A. M. Weiner, "Directly generated Gaussian-shaped optical frequency comb for microwave photonic filtering and picosecond pulse generation," IEEE Photon. Technol. Lett., vol. 24, no. 17, pp. 1484-1486, Sep. 1, 2012.

[7] A. R. C. Serrano, C. de Dios Fernandez, E. P. Cano, M. Ortsiefer, P. Meissner, and P. Acedo, "VCSEL-based optical frequency combs: Toward efficient single-device comb generation," IEEE Photon. Technol. Lett., vol. 25, no. 20, pp. 1981-1984, Oct. 15, 2013.

[8] R. Wu, V. Torres-Company, D. E. Leaird, and A. M. Weiner, "Supercontinuum-based 10-GHz flat-topped optical frequency comb generation," Opt. Exp., vol. 21, no. 5, pp. 6045-6052, Mar. 2013.

[9] C. de Dios and H. Lamela, "Improvements to long-duration low-power gain-switching diode laser pulses using a highly nonlinear optical loop mirror: Theory and experiment," J. Lightw. Technol., vol. 29, no. 5, pp. 700-707, Mar. 1, 2011.

[10] R. Zhou, S. Latkowski, J. O'Carroll, R. Phelan, L. P. Barry, and P. Anandarajah, "40 Nm wavelength tunable gain-switched optical comb source," Opt. Exp., vol. 19, no. 26, pp. B415-B420, Dec. 2011.

[11] P. M. Anandarajah et al., "Generation of coherent multicarrier signals by gain switching of discrete mode lasers," IEEE Photon. J., vol. 3, no. 1, pp. 112-122, Feb. 2011. 